\title{
A twist in the tail of human endothelin
}

The X-ray crystal structure of endothelin reveals a peptide with a compact globular conformation that may well represent the active form of the molecule.

ENDOTHELIN, a small 22-amino-acid peptide produced by endothelial cells, was originally defined as one of the most potent vasoconstrictors of the smooth muscle sheath that lines the larger blood vessels. As such, endothelin is implicated in the regulation of vascular tone and has been suggested to be important in cardiovascular diseases such as hypertension, vasospasm and atherosclerosis. A paper in this month's Nature Structural Biology now presents the first crystal structure of endothelin, as determined by Janes and colleagues $^{1}$. Of the various endothelin structures that have been proposed, most based on NMR data, this new X-ray crystal structure is suggested to be the best candidate for the active, in vivo conformation of this important molecule.

The single layer of endothelial cells that lines our blood vessels has a more than purely mechanical role in the circulatory system. These cells, in response to various stimuli, produce factors that are involved in maintaining vascular homeostasis - endothelin and the endothelium-derived relaxing factor nitric oxide being among the best known.

There are three variants of endothelin (ET-1, ET-2 and ET-3), all of which are derived by proteolytic cleavage from a larger precursor protein. The small size of the endothelin peptide makes it an ideal candidate for structure determination by NMR. Nonetheless, there is no consensus conformation among the different solution structures, which may be due to the non-aqueous conditions used to obtain sufficiently high concentrations of peptide for analysis.

The X-ray crystal structure of endothelin, derived from aqueous solution, reveals that the peptide adopts a compact globular conformation. The $\mathrm{N}$ terminus forms an extended $\beta$-strand followed by a bulge and loop (residues 5-11) and the Cterminal portion of endothelin forms an

Also in this month's Nature Structural Biology: in vitro selection of an RNA that can bind a small aliphatic hydrophobe; a three-dimensional model of the Revbinding element of HIV-1; a new graphical representation for the secondary and tertiary structure of group I introns; comparing interleukin-4 NMR and crystal structures; kinetic partitioning during protein folding; engineering an allosteric activation site in glycogen phosphorylase; and conformational analysis of protein side chains. irregular $\alpha$-helix (residues 12-21). The four cysteine residues - absolutely conserved between the endothelins, and the related sarafotoxins and bibrotoxin form two disulphide bridges that hold the $\mathrm{N}$-terminal strand close to the proximal part of the helix (see figure). Sequence conservation in the $\mathrm{C}$-terminal region suggests that all three endothelins will form similar $\alpha$-helical tails. Most sequence variability is seen in the bulge region of the structure.

In the absence of a homologous crystal structure, various of the NMR models were used as search structures; the backbone of the N-terminal portion of one of the NMR models was sufficiently similar to the same portion of the peptide in the crystal structure to allow a molecular replacement solution; indeed, the rootmean-square deviation (r.m.s.d.) between the two structures in this region is $2.0 \AA$. But when all the sequences are included in the comparison, the r.m.s.d. shoots up to $5.0 \AA$, emphasizing the very different C-terminal conformations.

There are two classes of endothelin receptor $\left(\mathrm{ET}_{\mathrm{A}}\right.$ and $\left.\mathrm{ET}_{\mathrm{B}}\right)$ with different affinities for the different endothelins: $\mathrm{ET}_{\mathrm{A}}$ has a similar affinity for ET-1 and ET-2 but a lower affinity for ET-3, whereas $\mathrm{ET}_{\mathrm{B}}$ has comparable affinity for all three endothelins. For the $\mathrm{ET}_{\mathrm{A}}$ receptor, the nature of the residue at position 2 of endothelin and the overall structure of the head group, residues 8-13, are important for binding.

For the $\mathrm{ET}_{\mathrm{B}}$ receptor, on the other hand, determinants in the $\mathrm{C}$-terminal tail are critical in the interaction. The $\alpha$ helix itself may be important for $\mathrm{ET}_{\mathrm{B}}$ receptor binding because the binding/activity is particularly sensitive to replacement of residues positioned on the same side of the helix.

The conformation of such a small peptide could well be influenced by packing interactions in the crystal. Janes et al. note that two intermolecular hydrogen bonds may distort the conformation of the head group, particularly in the vicinity of residues 8 and 13, but there are no close contacts to the backbone residues of the C-terminal helix, so crystal packing forces may not be responsible for the difference between the NMR and crystal structures in this region.

What relevance does the crystal structure of endothelin have to the biologically active conformation? The two disulphide bridges and the extensive network of

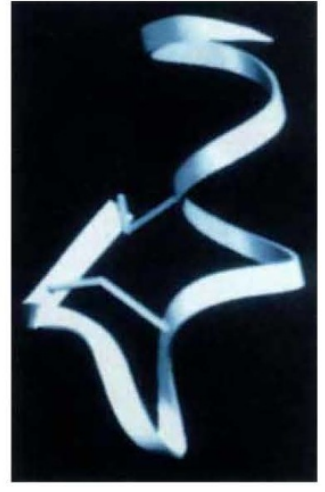

X-ray crystal struc ture of human endothelin ${ }^{1}$ (repro duced from ref. 1 with permission) The alpha-carbon ribbon is shown with the two disulphide bridges linking the distorted $\alpha$-helix with the $\mathrm{N}$-termina $\beta$-strand.

hydrogen bonds that stabilize the structure suggest that the peptide is unlikely to be very flexible and that the structure seen in the crystal may well represent the active conformation. Janes et al. point out that many of the invariant residues constitute the hydrophobic core of the structure and that variable residues can be accommodated without serious disruption of the structure.

What of the in vivo function of endothelin? Its role as a vasoconstrictor has recently been questioned by the finding that the blood pressure of transgenic 'knock-out' mice with only one copy of the gene, is slightly raised, rather than lowered as expected. Perhaps even more surprisingly, disruption of both copies of the gene results in an embryonic lethal phenotype with malformed craniofacial organs, which seems to originate from abnormalities of the pharnygeal arches ${ }^{2}$.

The endothelin knock-out mice clearly indicate that our understanding of the function of this small peptide, both in the control of vascular tone and during embryonic development, is far from complete. The insights gleaned from the X-ray crystallographic study ${ }^{1}$ should facilitate the design of reagents that modulate the activity of the endothelin receptors, the proteinases responsible for the processing reaction, and so on. Such reagents will be invaluable in further defining the complex role of endothelin in vivo. The next step is to determine the details of the interaction between peptide and receptor in the hope of bringing the structure closer towards what is still a somewhat elusive function.

Guy Riddihough

Guy Riddihough is Editor of Nature Structural Biology.

1. Janes, R.W., Peapus, D.H. \& Wallace, B.A. Nature struct. Biol. 1, 311-319 (1994)

2. Kurihara, Y. et al. Nature 368, 703-710 (1994) 